\title{
A very-low-protein diet ameliorates advanced diabetic nephropathy through autophagy induction by suppression of the mTORC1 pathway in Wistar fatty rats, an animal model of type 2 diabetes and obesity
}

\author{
Munehiro Kitada $^{1,2} \cdot$ Yoshio Ogura $^{2} \cdot$ Taeko Suzuki $^{2} \cdot$ Shi Sen $^{1,2} \cdot$ Seon Myeong Lee ${ }^{1,2}$. \\ Keizo Kanasaki $^{1,2} \cdot$ Shinji Kume ${ }^{3} \cdot$ Daisuke Koya $^{1,2}$
}

Received: 26 January 2016 / Accepted: 22 February 2016 / Published online: 28 March 2016

(C) Springer-Verlag Berlin Heidelberg 2016

\begin{abstract}
Aims/hypothesis The efficacy of a low-protein diet (LPD) on diabetic nephropathy is controversial. We aimed to investigate the renoprotective effects of an LPD and the underlying molecular mechanism in a rat model of type 2 diabetes and obesity.

Methods Diabetic male Wistar fatty ( $f a / f a$ ) rats (WFRs) were treated with a standard diet $(23.84 \%$ protein $)$ or an LPD (5.77\% protein) for 20 weeks from 24 weeks of age. We investigated the effect of the LPD on renal function, fibrosis, tubular cell damage, inflammation, mitochondrial morphology of proximal tubular cells (PTCs), apoptosis, autophagy and activation of mammalian target of rapamycin complex 1 (mTORC1).

Results Kidney weight, albuminuria, excretion of urinary liver-type fatty acid binding protein, levels of plasma cystatin $\mathrm{C}$ and changes in renal histology, including fibrosis, tubular
\end{abstract}

Electronic supplementary material The online version of this article (doi:10.1007/s00125-016-3925-4) contains peer-reviewed but unedited supplementary material, which is available to authorised users.

Munehiro Kitada

kitta@kanazawa-med.ac.jp

$\triangle$ Daisuke Koya

koya0516@kanazawa-med.ac.jp

1 Division of Anticipatory Molecular Food Science and Technology, Medical Research Institute, Kanazawa Medical University,

Uchinada, Ishikawa, Japan

2 Department of Diabetology and Endocrinology, Kanazawa Medical University, Uchinada, Ishikawa, Japan 920-0293

3 Department of Medicine, Shiga University of Medical Science, Ōtsu, Shiga, Japan cell damage and inflammation, were aggravated in WFRs compared with non-diabetic Wistar lean rats (WLRs). Fragmented and swelling mitochondria accumulated in PTCs and apoptosis were enhanced in the kidney of WFRs. Immunohistochemical staining of p62 and p-S6 ribosomal protein (p-S6RP) in the tubular lesions of WFRs was increased compared with WLRs. The LPD intervention clearly ameliorated damage as shown by the assessment of renal function and histology, particularly tubulointerstitial damage in diabetic kidneys. Additionally, the 5.77\% LPD, but not the $11.46 \%$ LPD, significantly suppressed p-S6RP levels and increased microtubule-associated protein light chain 3-II levels in the renal cortex. The LPD intervention partially decreased $\mathrm{HbA}_{1 \mathrm{c}}$ levels in WFRs, and no differences in mean BP were observed among any of the groups.

Conclusions/interpretation A very-low-protein diet improved advanced diabetic renal injuries, including tubulointerstitial damage, by restoring autophagy through the suppression of the mTORC1 pathway.

Keywords Autophagy · Diabetic nephropathy $\cdot$ Low-protein diet $\cdot$ Mammalian target of rapamycin complex $1 \cdot$ Wistar fatty rat

Abbreviations
$\begin{array}{ll}\text { FGF21 } & \text { Fibroblast growth factor 21 } \\ \text { GFP } & \text { Green fluorescent protein } \\ \text { HFD } & \text { High-fat diet } \\ \text { IPGTT } & \text { Intraperitoneal glucose tolerance test } \\ \text { IPITT } & \text { Intraperitoneal insulin tolerance test } \\ \text { KIM-1 } & \text { Kidney injury molecule-1 } \\ \text { L-FABP } & \text { Liver-type fatty acid binding protein } \\ \text { LC3 } & \text { Microtubule-associated protein light chain } 3\end{array}$




$\begin{array}{ll}\text { LPD } & \text { Low-protein diet } \\ \text { MCP-1 } & \text { Monocyte chemotactic protein 1 } \\ \text { MT } & \text { Masson's trichrome } \\ \text { mTORC1 } & \text { Mammalian target of rapamycin complex 1 } \\ \text { PAS } & \text { Periodic acid-Schiff } \\ \text { PTCs } & \text { Proximal tubular cells } \\ \text { S6RP } & \text { S6 ribosomal protein } \\ \text { STD } & \text { Standard diet } \\ \text { TLR2 } & \text { Toll-like receptor 2 } \\ \text { VLPD } & \text { Very-low-protein diet } \\ \text { WFRs } & \text { Wistar fatty rats } \\ \text { WLRs } & \text { Wistar lean rats }\end{array}$

\section{Introduction}

Diabetic nephropathy develops in $40 \%$ of patients with diabetes and remains the leading cause of end-stage kidney disease worldwide [1]. Multifactorial management, including diet therapy and glycaemic, BP and lipid control, is recommended for diabetic nephropathy. Regarding diet therapy, the efficacy of a low-protein diet (LPD) for advanced diabetic nephropathy remains controversial [2]. Previous clinical studies have not consistently shown beneficial effects of an LPD for the preservation of renal function in diabetic nephropathy [3-8]. However, other studies have shown that an LPD has beneficial effects in slowing the progressive decline in renal function [9, 10]. Nezu et al showed that an LPD for diabetic nephropathy improves the estimated glomerular filtration rate when patients adhere to a protein-restricted diet [11]. However, LPD adherence is often poor [3,11], which has contributed to the controversial results of previous clinical studies. In addition, the amount of protein restriction would be expected to be important for renoprotection. Ideura et al reported that a very-low-protein diet (VLPD) consisting of less than $0.5 \mathrm{~g} \mathrm{~kg}^{-1} \mathrm{day}^{-1}$ in the absence of malnutrition significantly suppressed renal dysfunction in patients with chronic glomerular nephritis who had serum creatinine levels of more than $530.6 \mu \mathrm{mol} / 1$ [12]. Thus, an LPD, particularly a VLPD without malnutrition, should have beneficial effects on advanced diabetic nephropathy as well as on chronic glomerular nephritis. Furthermore, regarding the mechanisms underlying the renoprotective effects of an LPD, data from previous animal experiments have shown that the main effects of an LPD might be to prevent the onset of diabetic nephropathy by improving abnormal metabolic factors and haemodynamics through multiple mechanisms, including improvements in glomerular hypertension, capillary resistance and hypertrophy [13-17]. The degree of tubulointerstitial damage rather than glomerular damage is predictively related to renal function decline [18-20]. However, the detailed molecular mechanisms underlying the effect of an LPD on renal tubular cells remain unclear. Furthermore, it has never been reported whether interventional treatment with an LPD improves advanced diabetic nephropathy.

Autophagy is a lysosomal degradation pathway that removes protein aggregates and damaged or excess organelles such as mitochondria, thereby contributing to the maintenance of intracellular homeostasis under various stress conditions [21]. Impairment of autophagy is observed in renal cells, including tubular cells, and is implicated in the pathogenesis of kidney diseases, including diabetic nephropathy. Therefore, improvement of autophagy should be the therapeutic target for diabetic nephropathy. Nutrient-sensing pathways that depend on extracellular nutrient conditions are recognised as factors that regulate autophagy $[22,23]$. The mammalian target of rapamycin complex 1 (mTORC1) pathway is activated by a state of overnutrition, resulting in the suppression of autophagy. Previous reports have shown that mTORC1 activity is increased in renal cells, including tubular cells, in the diabetic state [24-26]. Because amino acids are recognised as mTORC1 activators [27], an LPD, which involves amino acid restriction, should suppress the mTORC1 pathway. Therefore, we hypothesised that an LPD might activate autophagy by inhibiting the mTORC1 pathway in renal cells, particularly tubular cells, thereby leading to renoprotection during diabetic nephropathy. To examine this hypothesis, we characterised the effects of an interventional VLPD on advanced diabetic nephropathy in Wistar fatty ( $f a / f a$ ) rats (WFRs), an animal model of type 2 diabetes and obesity.

\section{Methods}

Materials For details regarding materials, please refer to the electronic supplementary material (ESM) Methods.

Experimental animals Male and female Wistar lean $(f a /+)$ rats (WLRs) were provided by the Takeda Pharmaceutical Company Biological Institute (Osaka, Japan) [28] and maintained at Kanazawa Medical University. Male diabetic WFRs and age-matched non-diabetic WLRs were used. At 24 weeks of age, the rats were divided into four groups: (1) WLRs fed a standard diet (STD); (2) WLRs fed an LPD; (3) WFRs fed an STD; and (4) WFRs fed an LPD. The STD comprised 23.84\% protein, $16.80 \%$ fat, $59.36 \%$ carbohydrates and $14.85 \mathrm{~kJ} / \mathrm{g}$ energy. The LPD comprised $5.77 \%$ protein, $16.48 \%$ fat, $77.75 \%$ carbohydrates and $14.81 \mathrm{~kJ} / \mathrm{g}$ energy. The dietary intervention was performed for 20 weeks. We also used chow comprising $11.46 \%$ protein, $16.58 \%$ fat, $71.97 \%$ carbohydrates and $14.81 \mathrm{~kJ} / \mathrm{g}$ energy for some experiments, and some WFRs were fed $30 \%$ calorie-restricted chow during the experimental period.

The Research Center for Animal Life Science of Kanazawa Medical University approved all experiments, and all 
experiments were performed in accordance with relevant guidelines and regulation (for further details, see ESM Methods.).

Biochemical measurements $\mathrm{HbA}_{1 \mathrm{c}}$ levels were measured using a DCA 2000 Analyzer (Siemens Medical Solutions Diagnostics, Tokyo, Japan) at the end of the experiment. An intraperitoneal glucose tolerance test (IPGTT) and an intraperitoneal insulin tolerance test (IPITT) were performed at 20 weeks of intervention, as previously described [29]. Urinary albumin and liver-type fatty acid binding protein (L-FABP), plasma cystatin $\mathrm{C}$, insulin and fibroblast growth factor 21 (FGF21) levels were measured using ELISA kits (see ESM Methods).

Morphological analysis and immunohistochemistry, western blot analysis and real-time PCR The kidney sections were stained with Masson's trichrome (MT)/periodic acid-Schiff (PAS) reagent, and immunohistochemical staining was performed using antibodies against CD68 (1:50), kidney injury molecule-1 (KIM-1) (1:100), p62 (1:100) and p-S6 ribosomal protein (S6RP) (1:100), as previously described [28, 30, 31]. (For further details of morphological and immunohistochemical analysis, see ESM Methods.) Western blotting was performed using antibodies to cleaved caspase-3 (1:1000), $\beta$ actin (1:1000), p-S6RP (1:1000), S6RP (1:1000), p-Akt (1:1000), Akt (1:1000) and microtubule-associated protein light chain 3 (LC3)-I/II (1:1000). The isolation of total RNA from the renal cortex, cDNA synthesis and quantitative realtime PCR were performed, as previously described [28]. TaqMan probes for type III collagen, CD68, IL-6, TNF- $\alpha$, monocyte chemotactic protein 1 (MCP-1) and toll-like receptor 2 (TLR2) were purchased from Thermo Fisher Scientific (Waltham, MA, USA). The analytical data were adjusted to the levels of 18S mRNA expression as an internal control.

Transmission electron microscopy Mitochondrial morphology and fragmented mitochondria in the proximal tubular cells (PTCs) were observed using transmission electron microscopy, as previously described [28, 32]. (For further details regarding electron microscopic mitochondrial morphology, see ESM Methods.)

\footnotetext{
Monitoring autophagosomes in renal proximal tubular cells using LC3-green fluorescent protein transgenic mice Male LC3-green fluorescent protein (GFP) mice (C57BL/6 background) were obtained from Riken BioResource Center (Ibaraki, Japan). Twelve-week-old mice were fed an STD or a high-fat diet (HFD) for 4 weeks; a subset of HFD-fed mice were then switched to an LPD for 2 days. After $48 \mathrm{~h}$ of starvation, we determined the autophagy in PTCs, as previously described [33].
}

Statistical analysis Data are expressed as means \pm SD. ANOVA followed by Tukey's multiple comparison test was used to determine the significance of pairwise differences among three or more groups, and Student's $t$ test was used for unpaired comparisons. A $p$ value of $<0.05$ was considered significant.

\section{Results}

Characteristics of the experimental rats The whole-body, fat and kidney weights of the WFRs were significantly higher in comparison with other groups (Fig. 1a-c) after the 20 week intervention. The mean BP was not significantly changed in any of the groups (Fig. 1d). WFRs exhibited significantly elevated $\mathrm{HbA}_{1 \mathrm{c}}$ compared with WLRs, and the LPD induced a partial improvement in $\mathrm{HbA}_{1 \mathrm{c}}$ levels in WFRs after the intervention (Fig. 1e). Fasting plasma insulin levels were higher in WFRs compared with WLRs, and there were no differences between WFRs and LPD-fed WFRs (Fig. 1f). Mean food intake was $15.5 \%$ lower in LPD-fed WFRs compared with STD-fed WFRs during the experimental period (Fig. 1g). The results of the IPGTT showed no statistically significant difference between WFRs and LPD-fed WFRs (Fig. 1h, i). However, the AUC for the IPITT was significantly decreased in LPD-fed WFRs compared with STD-fed WFRs (Fig. 1j, k). Plasma fasting total cholesterol and triacylglycerol levels were also significantly elevated in WFRs compared with WLRs (Fig. 11, m) after the intervention. Increases in total cholesterol levels of WFRs were reduced by the LPD; however, the LPD did not lead to a statistically significant difference in the triacylglycerol levels of WFRs (Fig. 11, m). The plasma FGF21 concentration was measured after a $16 \mathrm{~h}$ fast and at postprandial times. No differences were observed in fasting FGF21 levels in the four groups. Although plasma FGF21 levels were markedly reduced after food intake in WLRs and STD-fed WFRs, these effects were not observed after food intake in LPD-fed WLRs and WFRs (Fig. 1n).

Changes in urinary albumin and L-FABP excretion and plasma cystatin C At 24 weeks of age, urinary albumin/ creatinine and L-FABP/creatinine ratios were both already higher in WFRs than in WLRs (Fig. 2a, b). Levels of urinary albumin and L-FABP excretion in WFRs were markedly increased compared with WLRs after the 20 week LPD intervention (Fig. 2a, b). The LPD clearly reduced the urinary albumin and L-FABP excretion levels in WFRs, but 30\% calorie restriction did not have the same effect (Fig. 2a, b). Additionally, urinary L-FABP excretion values were significantly improved after the 20 week LPD intervention compared with the values in 24week-old WFRs. However, urinary albumin excretion values after the LPD intervention remained at almost the same levels as those observed in 24-week-old WFRs. After the intervention, plasma cystatin C levels were also significantly higher in WFRs 

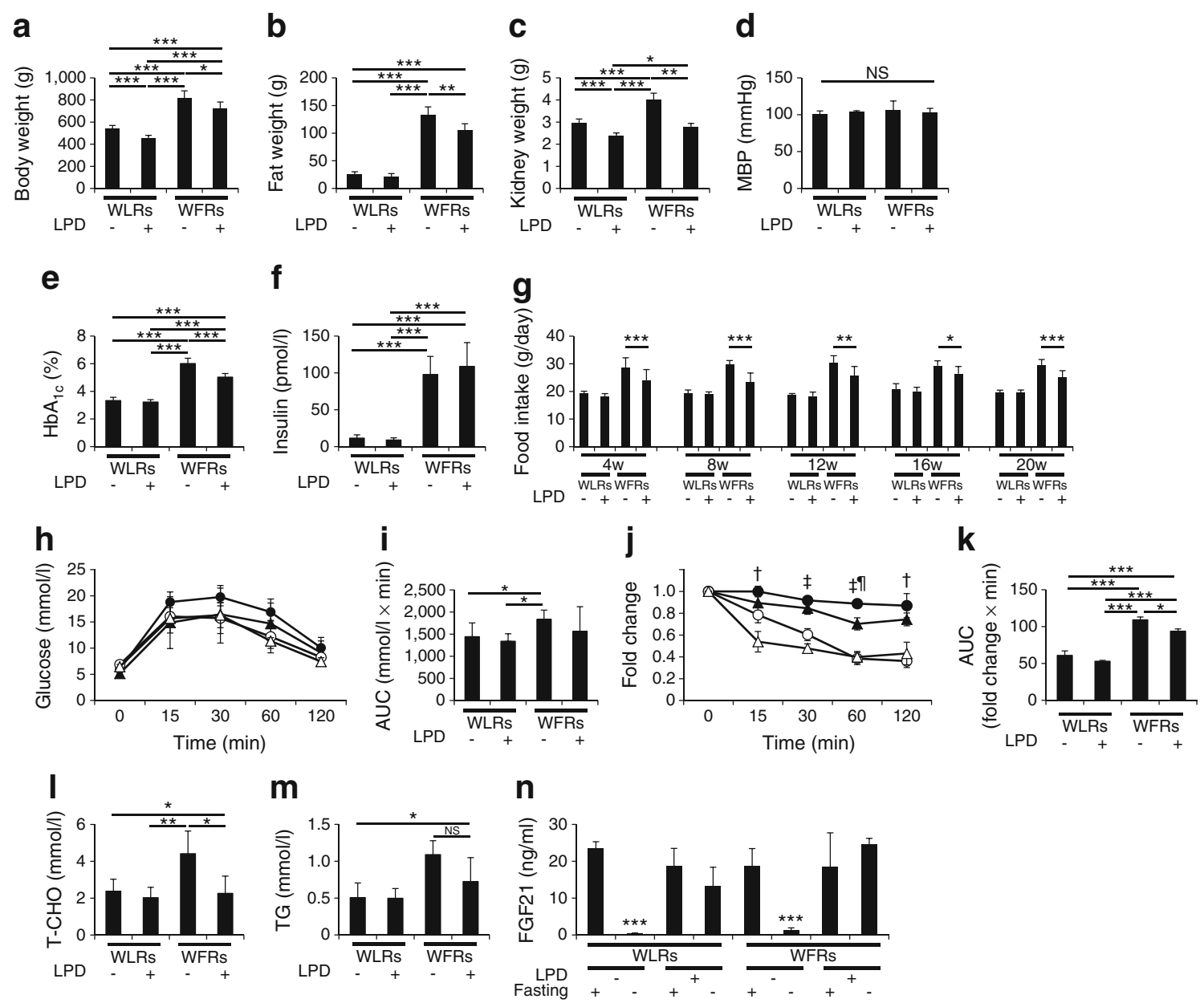

Fig. 1 Characteristics of rats. (a) Whole-body weight, (b) fat weight, (c) kidney weight and (d) mean BP (MBP) after the 20 week intervention $(n=7-9)$. (e) $\mathrm{HbA}_{1 \mathrm{c}}$ levels $(n=7-9)$ and (f) fasting plasma insulin levels ( $n=4-6)$ following the intervention. (g) Food intake during the experimental periods $(n=7-9)$. (h) Data from the IPGTT and (j) IPITT, and (i) AUCs for the IPGTT and (k) IPITT after the intervention $(n=3)$. (l) Levels of fasting total cholesterol (T-CHO) $(n=4-6),(\mathbf{m})$ triacylglycerol (TG) $(n=4-6)$ and (n) fasting or postprandial plasma FGF21 after the

intervention $(n=3-4)$. Data shown are means $\pm \mathrm{SD} .{ }^{*} p<0.05$, ${ }^{* *} p<0.01,{ }^{* * *} p<0.001$ vs the indicated groups. White circles, WLRs; white triangles, WLRs + LPD; black circles, WFRs; black triangles, WFRs + LPD. ${ }^{\dagger} p<0.05$ vs WLRs and WLRs + LPD; ${ }^{\star} p<0.01$ vs WLRs and WLRs + LPD; ${ }^{\natural} p<0.05$ vs WFRs + LPD; W, week. To convert values for $\mathrm{HbA}_{1 \mathrm{c}}$ in $\mathrm{DCCT} \%$ into $\mathrm{mmol} / \mathrm{mol}$, subtract 2.15 and multiply by 10.929

than in WLRs, and LPD intervention restored these levels in WFRs (Fig. 2c).

Changes in renal fibrosis and tubular cell damage Representative photographs of whole kidneys and

photomicrographs of MT-stained kidney sections from 24week-old rats showed renal hypertrophy in WFRs (Fig. 3a). The renal fibrosis observed using MT staining and the mRNA expression of type III collagen were shown to be higher in the kidneys of WFRs compared with WLRs at this time (Fig. 3b-

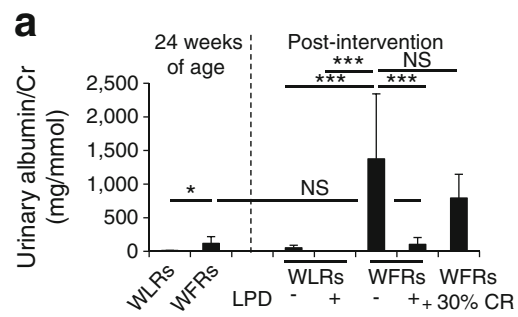

Fig. 2 Changes in renal function of rats. (a) Urinary albumin/creatinine (Cr) ratio and (b) $\mathrm{L}-\mathrm{FABP} / \mathrm{Cr}$ ratio at 24 weeks of age and after the intervention ( $n=7-9)$ (WFRs $+30 \%$ calorie restriction [CR], $n=3)$. (c)
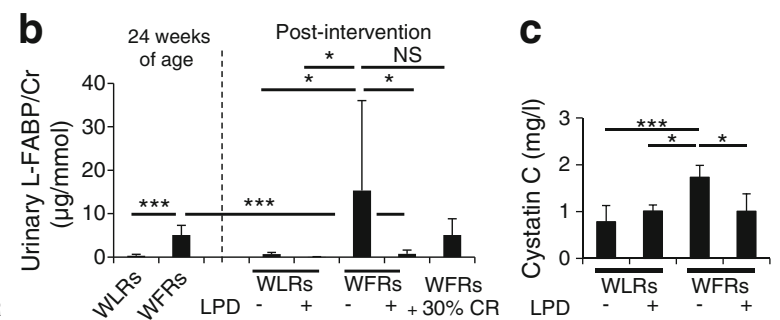

Plasma cystatin $C$ levels after the intervention $(n=7-9)$. Data shown are means \pm SD. ${ }^{*} p<0.05, * * * p<0.001$ vs indicated groups 
a
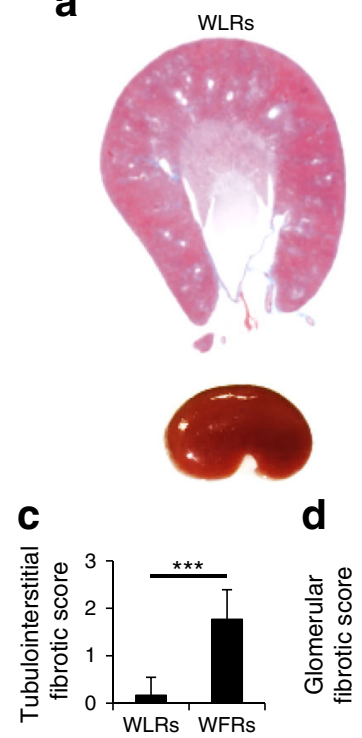

f

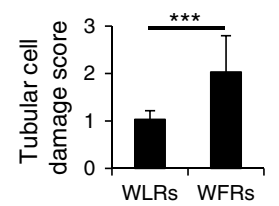

d

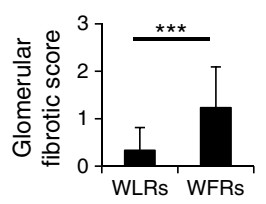

$\mathbf{g}$

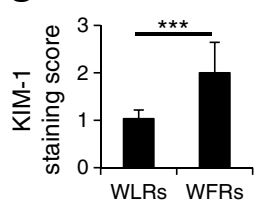

WFRs

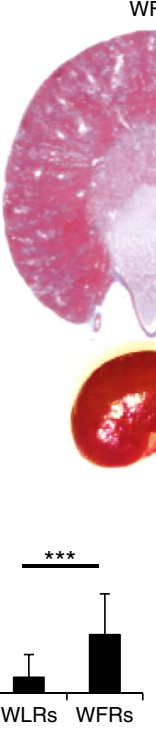

e

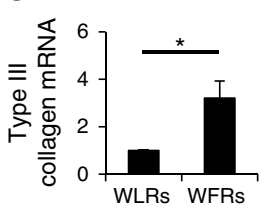

h

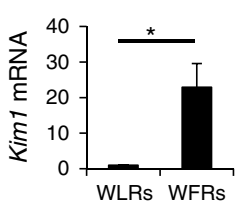

b

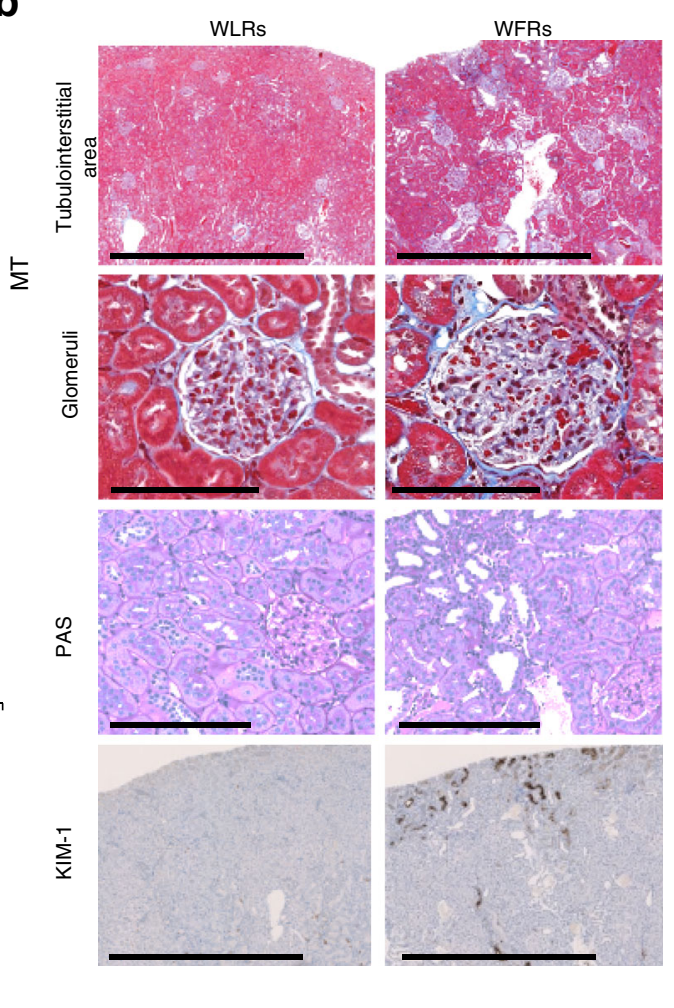

Fig. 3 Changes in renal fibrosis and tubular cell damage at 24 weeks of age. (a) Representative photographs of whole kidneys and MT-stained $(\times 4)$ kidney sections. (b) MT staining of tubulointerstitial area (scale bars, $1 \mathrm{~mm}$ ) and glomeruli (scale bars, $100 \mu \mathrm{m}$ ), PAS staining for the evaluation of tubular cell damage (scale bars, $200 \mu \mathrm{m}$ ) and immunohistochemistry for KIM-1 (scale bars, $1 \mathrm{~mm}$ ). (c) Tubulointerstitial scores $(n=3)$ and (d) glomerular fibrotic scores, obtained using MT staining. (e) mRNA

e). The tubular cell damage scores evaluated by PAS staining and KIM-1 immunohistochemical staining and mRNA expression were also already significantly higher in WFRs than in WLRs (Fig. 3b, f-h). After the 20 week LPD intervention, the kidneys of WFRs were markedly larger than those of WLRs (Fig. 4a), and renal fibrosis and tubular cell damage were consistently increased in the kidneys of WFRs compared with WLRs (Fig. 4b-h). LPD intervention clearly improved all these advanced renal injuries in WFRs.

Changes in inflammation in the kidney CD68 immunohistochemical staining scores in tubulointerstitial lesions and mRNA expression inflammation-related genes including Cd68, Mcpl (also known as Ccl2), Tnfa (also known as Tnf) and $T l r 2$ in the renal cortex were already significantly higher in WFRs compared with WLRs in 24-week-old rats (Fig. 5a-f). After the 20 week experimental period, renal inflammation, as indicated by CD68 immunohistochemical staining and mRNA expression of $C d 68, M c p 1, T n f a, T l r 2$ and $I l 6$, was consistently aggravated in the renal cortex of WFRs compared with WLRs (Fig. $5 \mathrm{~g}-\mathrm{m}$ ). However, LPD intervention almost completely abrogated the renal inflammation in WFRs. expression of type III collagen adjusted to $18 \mathrm{~S}$ levels in the renal cortex at 24 weeks of age $(n=3)$. (f) Tubular cell damage scores obtained using PAS staining $(n=3)$. (g) Semi-quantification of KIM-1 staining scores $(n=3)$. (h) mRNA expression of Kim1 normalised to $18 \mathrm{~S}$ levels in the renal cortex $(n=3)$. Data shown are means \pm SD. ${ }^{*} p<0.05,{ }^{* * *} p<0.001$ vs other groups

Alterations in mitochondrial morphology in PTCs, glomerular basement membrane and podocytes, and apoptosis in the kidney Mitochondrial morphology was altered in PTCs of WFRs: fragmented mitochondria, mitochondrial swelling and increased numbers of PTCs without elongated mitochondria were observed (Fig. 6a, b). The LPD improved the alterations in mitochondrial morphology, and autophagosomes were observed in LPD-fed WFRs (Fig. 6a). The thickness of the glomerular basement membrane was markedly increased in WFRs, and podocyte foot process effacement was observed in the glomeruli of WFRs. The changes in glomerular basement membrane and podocyte foot process in WFRs were corrected by LPD treatment (Fig. 6c). Additionally, apoptosis, as determined by cleaved caspase 3 content, was increased in the renal cortex of WFRs, and the LPD improved the apoptosis in WFRs (Fig. 6d, e).

Changes in autophagy and mTORC1 pathway activation in the kidney Immunohistochemical staining for p62 was significantly enhanced in both tubular lesions and glomerular podocytes of WFRs, and the LPD ameliorated p62 staining intensity in the kidneys of WFRs (Fig. 7a, b). 
Fig. 4 Changes in renal fibrosis and tubular cell damage after the LPD intervention. (a)

Representative photographs of whole kidneys and MT-stained (×4) kidney sections. (b) MT staining of tubulointerstitial area (scale bars, $1 \mathrm{~mm}$ ) and glomeruli (scale bar, $100 \mu \mathrm{m}$ ), PAS staining for the evaluation of tubular cell damage (scale bars, $200 \mu \mathrm{m}$ ) and immunohistochemistry for KIM1 (scale bars, $1 \mathrm{~mm}$ ). (c) Tubulointerstitial scores $(n=3)$, and (d) glomerular fibrotic scores $(n=3)$, obtained using MT staining. (e) mRNA expression of type 3 collagen adjusted to $18 \mathrm{~S}$ levels in the renal cortex $(n=3)$. (f) Tubular cell damage scores obtained using PAS staining $(n=3)$. (g) Semi-quantification of KIM-1 staining scores $(n=3)$. (h) mRNA expression of $\mathrm{Kim} 1$ normalised to $18 \mathrm{~S}$ levels in the renal cortex $(n=7-9)$. Data shown are means $\pm \mathrm{SD}$. $* * * p<0.001$ vs other groups a
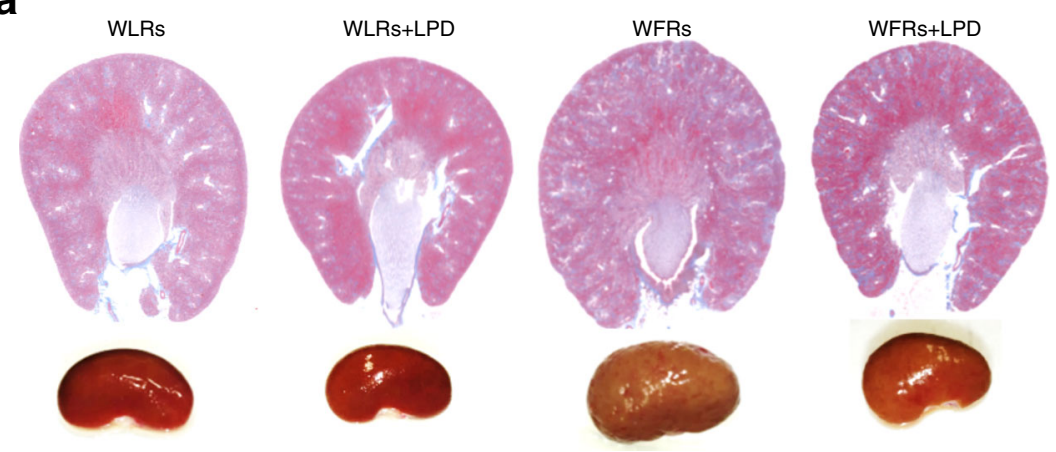

\section{b}
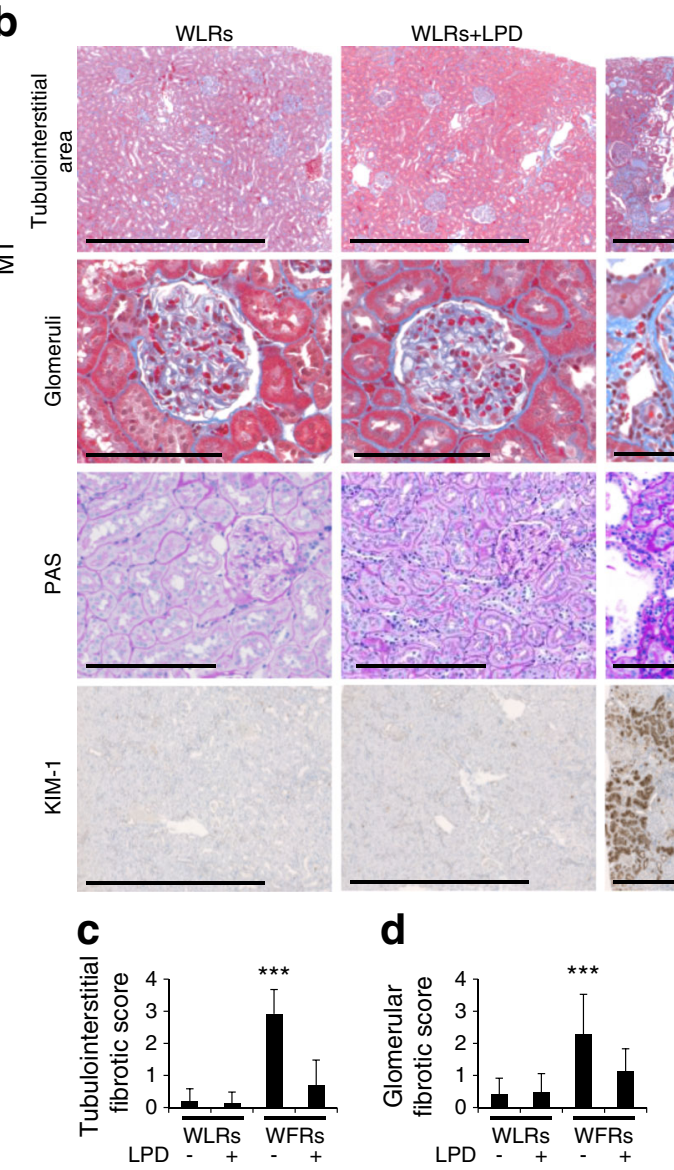

WFRs

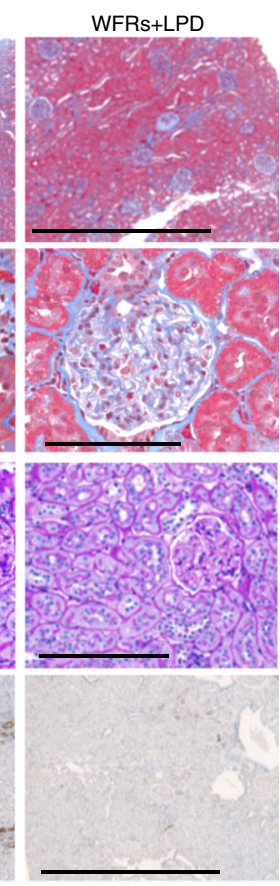

e
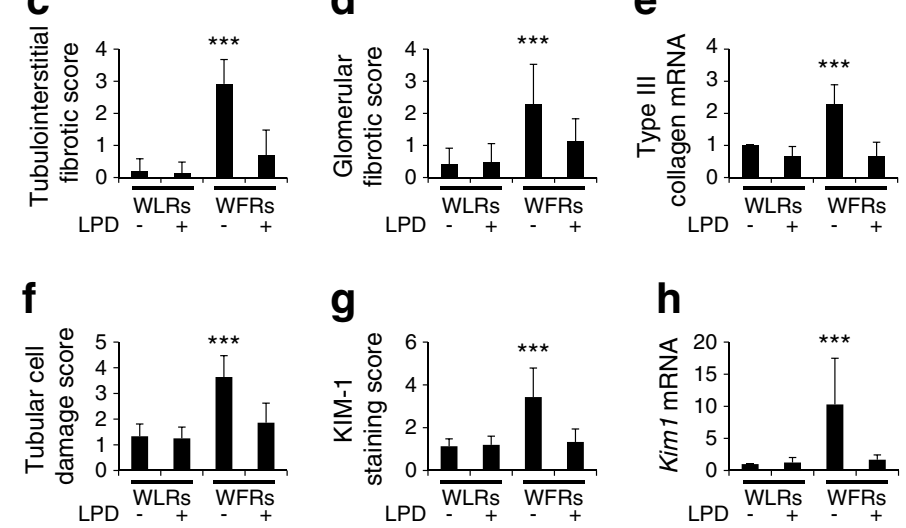

g

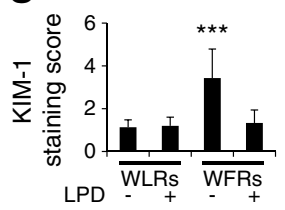

Immunohistochemical staining for p-S6RP (a downstream molecule of mTORC1) was also increased in both tubular lesions and glomerular podocytes of WFRs, and LPD decreased the staining to a level similar to that observed in WLRs (Fig. 7a, c). Levels of p-S6RP were increased in the renal cortex of WFRs compared with WLRs, but were significantly decreased after switching the diet from STD to $5.77 \%$ LPD for as little as 2 weeks (Fig. 7d, e). In addition, there was no difference in p-Akt protein levels between STDand LPD-fed WFRs (Fig. 7d, f). LC3-II levels were also significantly increased by the LPD, indicating that LPD induced autophagy in the renal cortex (Fig. $7 \mathrm{~d}$, g). However, the 
Fig. 5 Changes in renal inflammation. Representative photographs of CD68

immunohistochemistry (scale bar, $1 \mathrm{~mm}$ ) at (a) 24 weeks of age and (g) after the intervention. (b) Semi-quantification of CD68 staining scores at 24 weeks of age $(n=3)$ and (h) after the intervention $(n=3)$. (c) mRNA expression of $C d 68$, (d) $M c p 1,(\mathbf{e})$ Tnfa and (f) Tlr2, normalised to $18 \mathrm{~S}$ mRNA expression, in the renal cortex at 24 weeks of age $(n=3)$. (i) mRNA expression of Cd68, (j) Mcpl, (k) Tnfa, (l) Il6 and (m) Tlr2, normalised to $18 \mathrm{~S}$ mRNA expression, in the renal cortex after the intervention $(n=7-9)$. Data shown are means \pm SD. ${ }^{*} p<0.05,{ }^{* * *} p<0.001$ vs other groups
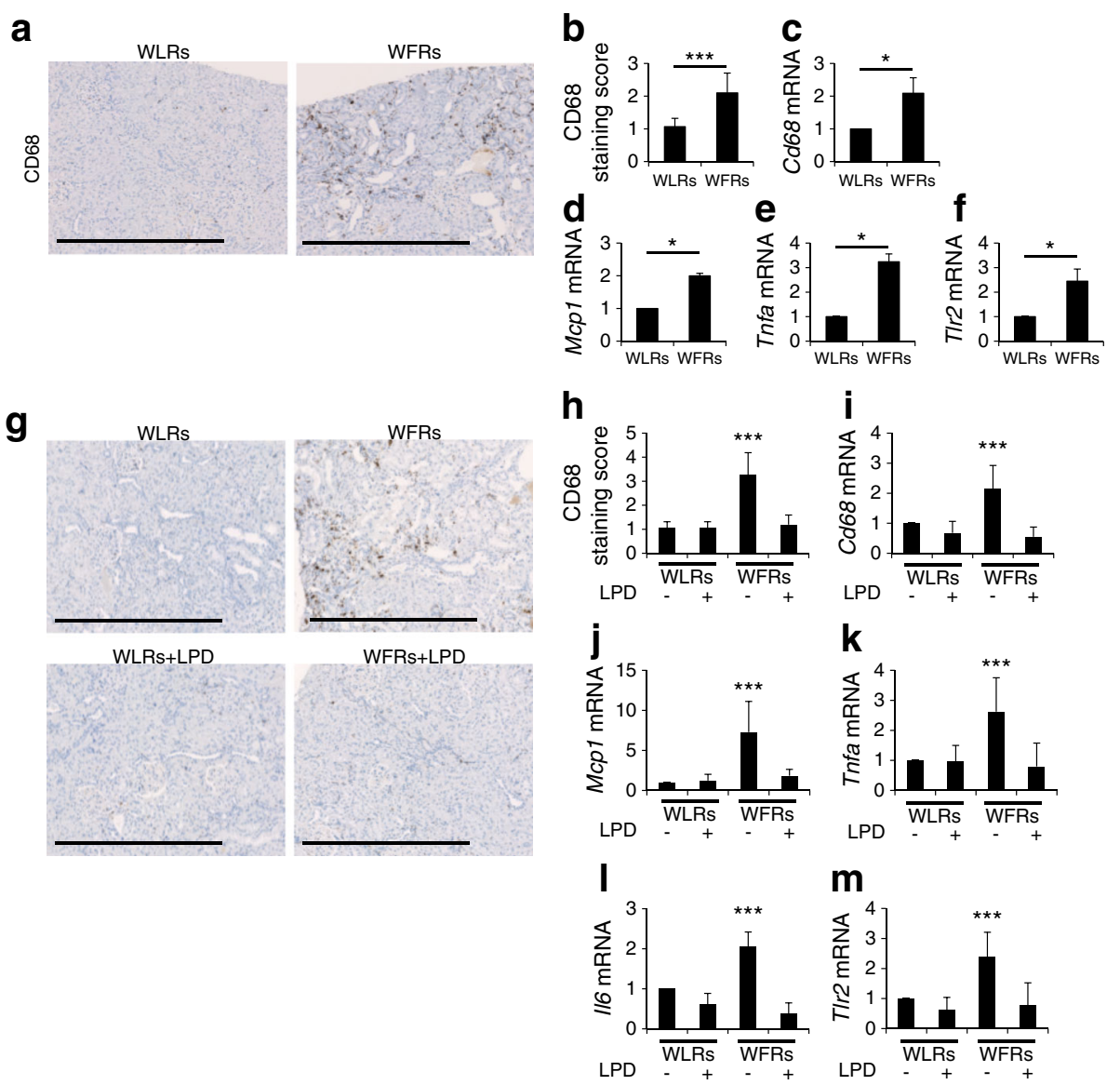

$11.46 \%$ LPD did not induce suppression of p-S6RP or increase in LC3-II levels in the kidneys (Fig. 7h-j).

In addition, mice fed an HFD for 4 weeks showed a decrease in $48 \mathrm{~h}$ starvation-induced autophagy in PTCs compared with mice fed an STD. However, in these mice, switching from HFD to LPD for 2 days restored the starvation-induced autophagy in PTCs. Thus, the LPD induced autophagy in the PTCs of LC3-GFP transgenic mice (Fig. 7k).

\section{Discussion}

In this study we demonstrated that dietary intervention with an LPD dramatically improved advanced diabetes-induced renal injuries by restoring autophagy through the suppression of mTORC1.

LPD intervention clearly suppressed the increase in urinary albumin and L-FABP excretion and decline in renal function in WFRs. Furthermore, LPD intervention led to improvement in all indicators of renal injury during the progression of diabetic nephropathy, particularly in tubulointerstitial lesions. In addition, we evaluated the effect of $30 \%$ calorie restriction in WFRs to estimate the effect of decreased food intake on diabetic nephropathy, because the mean food intake in LPD-fed
WFRs was $15.5 \%$ less than that of STD-fed WFRs during the experimental period. Both urinary albumin and L-FABP excretion in calorie-restricted WFRs were partially reduced, but no statistically significant difference was observed compared with STD-fed WFRs. $\mathrm{HbA}_{1 \mathrm{c}}$ levels in 30\% calorie-restricted WFRs were $5.4 \pm 0.26 \%(35.52 \pm 2.89 \mathrm{mmol} / \mathrm{mol})$, a level that was significantly lower than those of STD-fed WFRs and not significantly different from LPD-fed WFRs. Increased levels of urinary albumin and L-FABP excretion were almost completely prevented in LPD-fed WFRs, whereas $\mathrm{HbA}_{1 \mathrm{c}}$ levels in both calorie-restricted WFRs and LPD-fed WFRs were only moderately reduced. Furthermore, urinary LFABP excretion in WFRs, which was already elevated at 24 weeks of age, significantly improved after LPD intervention, and LPD intervention inhibited the progression to higher levels of albuminuria in WFRs after 20 weeks of intervention. Thus, although dietary therapy for diabetes basically involves calorie restriction, LPD intervention might provide a greater renoprotective effect, particularly tubulointerstitial protection, in advanced diabetic nephropathy, compared with calorie restriction, and this effect might be independent of glucose levels. Because tubulointerstitial injury is important in the progression of kidney diseases, including diabetic nephropathy, our data showing that LPD improved tubulointerstitial 
Fig. 6 Morphology of mitochondria in PTCs, glomerular basement membrane (GBM) and podocyte foot process, and apoptosis. (a) Representative transmission electron microscopy images of PTCs (scale bar, $500 \mathrm{~nm}$ ). The arrows indicate fragmented mitochondria, and the arrowheads indicate swollen mitochondria; autophagosomes are also shown. (b) The ratio of PTCs without elongation of mitochondria to total PTCs. (c) Representative transmission electron microscopy images of GBM thickness and podocyte foot process effacement (scale bar, $2 \mu \mathrm{m}$ ). The arrowheads indicate GBM thickness, and the arrows indicate podocyte foot process effacement. (d)

Representative western blots of cleaved caspase 3 content and (e) the quantitative ratios of cleaved caspase 3 to $\beta$-actin in the renal cortex $(n=3)$. Data shown are means \pm SD. $* * * p<0.001 \mathrm{vs}$ other groups
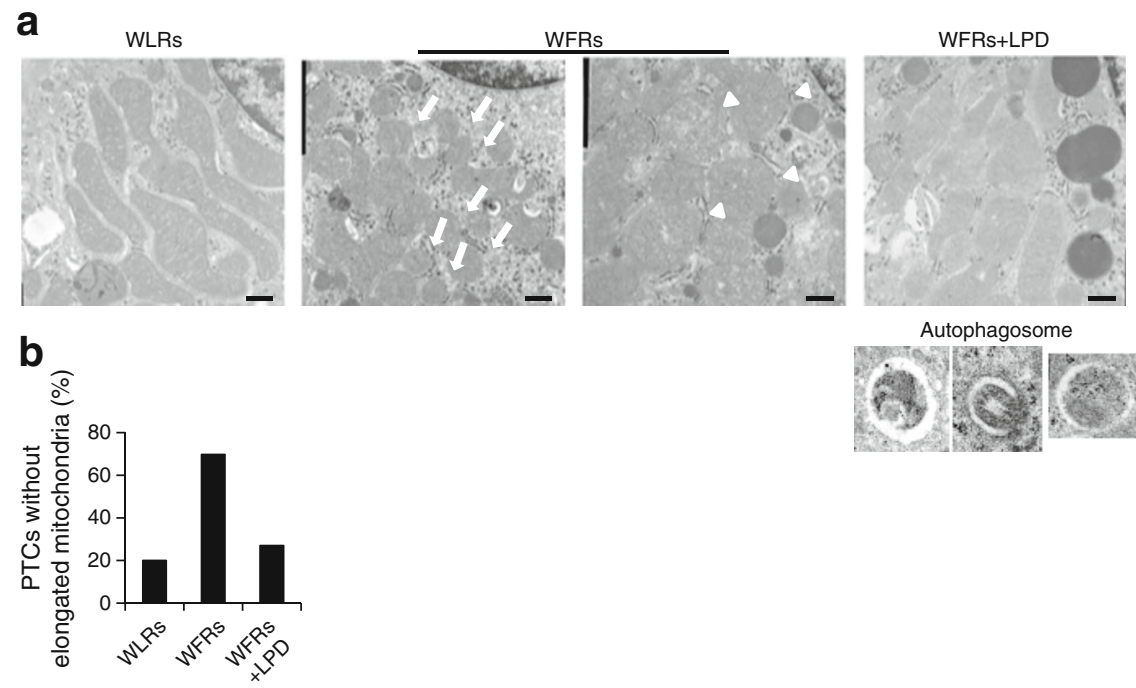

C
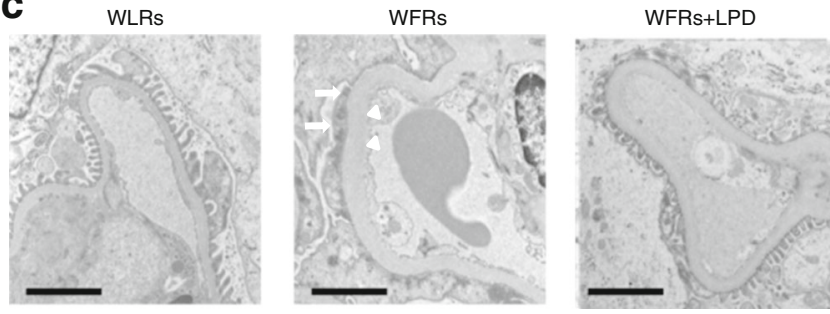

d

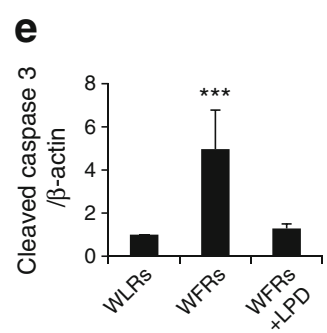

injury in advanced diabetic nephropathy should have a strong impact.

Autophagy plays a crucial role in maintaining mitochondrial quality through the lysosomal degradation of damaged mitochondria under various stress conditions [34-36]. Therefore, impaired autophagy contributes to the accumulation of damaged mitochondria, resulting in increased mitochondrial oxidative stress, inflammation and apoptosis. In renal tubular cells, basal autophagy activity is less than that in other renal cells, such as glomerular podocytes [37]. However, autophagy in renal tubular cells is induced through cellular stresses, including hypoxia and proteinuria, and the activation of autophagy protects tubular cells from cellular dysfunction and apoptosis. Previous studies have shown that the impairment of autophagy is associated with the pathogenesis of kidney diseases, including HFD-induced renal dysfunction [30], ageing kidneys [31] and diabetic nephropathy [28]. In this study we showed that fragmented mitochondria accumulated in diabetic PTCs, possibly due to impaired autophagy, resulted in increased apoptosis. In addition to the effects in PTCs, podocyte abnormalities, including foot process effacement, were observed in WFRs. These abnormalities were also likely to be associated with the impairment of autophagy, as indicated by enhanced immunohistochemical staining for $\mathrm{p} 62$.

The mTORC1 pathway is recognised as an autophagy regulatory factor, and overnutrition-induced activation of the mTORC1 pathway suppresses autophagy [34]. Previously, Yamahara et al showed that obesity-mediated autophagy insufficiency exacerbates proteinuria-induced tubulointerstitial lesions by causing hyperactivation of mTORC1 in an HFDinduced obesity mouse model [30]. Therefore, the restoration of autophagy through the inhibition of mTORC1 activity in the kidney is a novel therapeutic target for renoprotection in diabetic nephropathy. mTORC1 activation is positively regulated by growth factors such as insulin. Amino acids are also recognised as mTORC1 stimulators, and their depletion strongly induces autophagy. Grumati et al showed that type VI collagen-deficient mice $\left(\mathrm{Col}_{6 \mathrm{al}} \mathrm{I}^{-/}\right)$with muscular dystrophies have impaired autophagy, resulting in myofibre degeneration associated with dysfunctional mitochondria and 
Fig. 7 Changes in autophagy and mTORC1 pathway in the kidney.

(a) Representative photographs of immunohistochemistry for $\mathrm{p} 62$ and p-S6RP in tubular lesions (scale bar, $200 \mu \mathrm{m}$ ) and glomeruli (scale bar, $100 \mu \mathrm{m})(n=3)$. (b) Semi-quantification of p62 and (c) p-S6RP staining scores in tubulointerstitial lesions $(n=3)$.

(d) Representative western blots of p-S6RP, S6RP,

p-Akt, Akt, LC3-I/II and $\beta$-actin in the renal cortex after switching from an STD to the $5.77 \%$ LPD for 2 weeks. (e) Quantitative ratios of p-S6RP to S6RP, (f) fold change of p-Akt and (g) LC3-II to those of STD-fed WLRs $(n=3)$.

(h) Representative western blots of p-S6RP, S6RP and LC3-I/II in the renal cortex after switching from an STD to the $11.46 \%$ LPD for 2 weeks. (i) Quantitative ratios of p-S6RP to S6RP and (j) levels of LC3-II $(n=3)$. Data shown are means \pm SD. $* p<0.05$,

*** $p<0.001$ vs the indicated groups $(\mathbf{e}, \mathbf{g})$ or vs the WLRs $(\mathbf{f}, \mathbf{i})$.

(k) LPD induces autophagy in PTCs of HFD-induced obese mice. Representative images of PTCs in the kidneys of LC3-GFP transgenic mice after $48 \mathrm{~h}$ starvation following: (1) an STD for 4 weeks; (2) an HFD for 4 weeks; (3) a $5.77 \%$ LPD for 2 days after HFD for 4 weeks. The arrows indicate the LC3GFP-positive puncta a

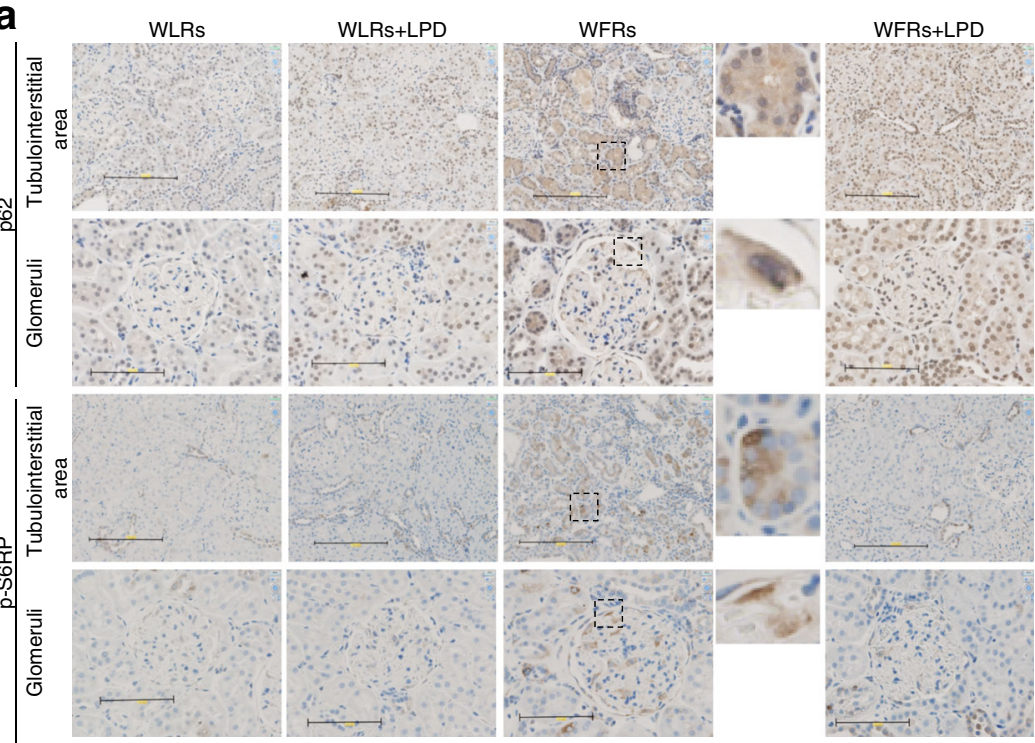

b

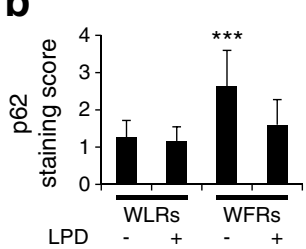

d

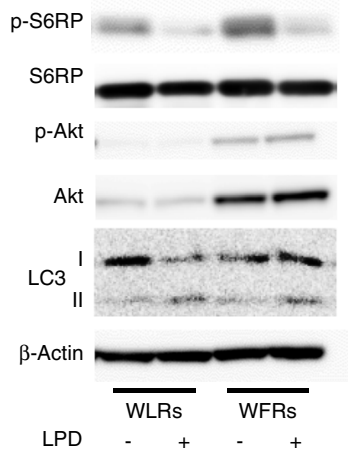

h

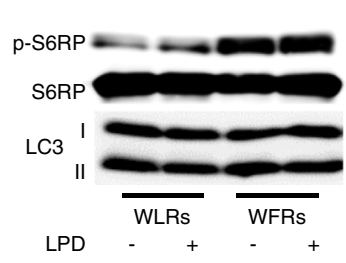

K (1) Standard Diet
+48 h starvation

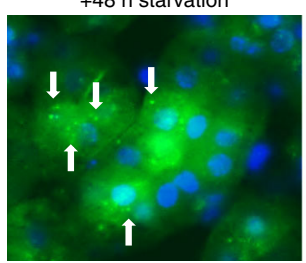

C

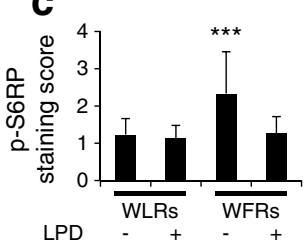

e

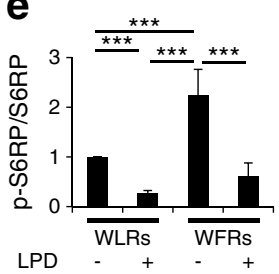

f

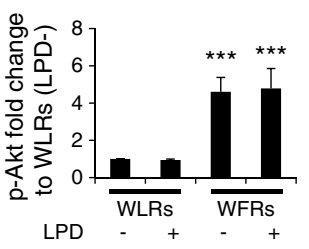

g

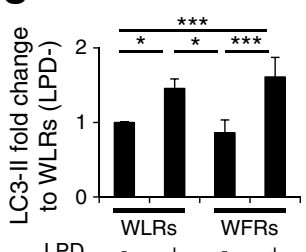

i
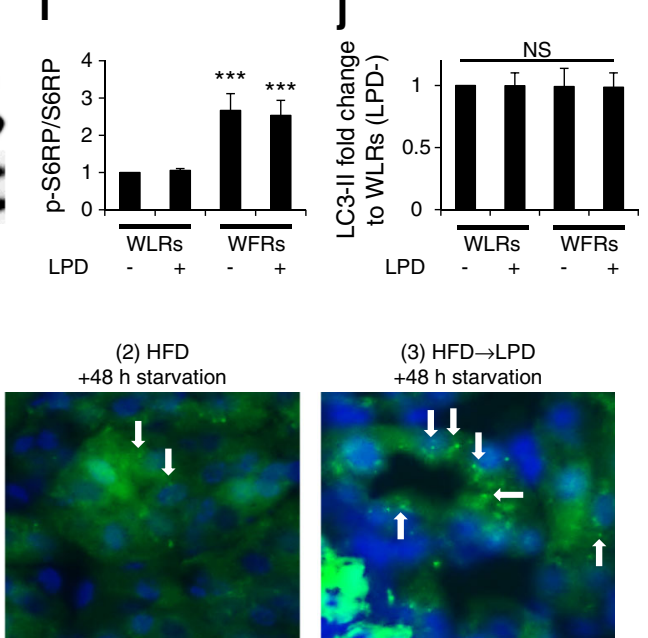

(3) HFD $\rightarrow$ LPD +48 h starvation

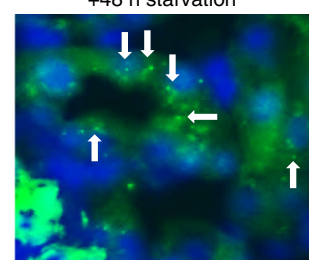


spontaneous apoptosis [38]. An LPD (5\%) restored myofibre survival and ameliorated the dystrophic phenotype in Coloal $1^{-/}$mice by inducing autophagy, which removed the structurally abnormal mitochondria in a manner similar to that of rapamycin, an mTOR inhibitor. Similarly, we showed that a 5.77\% LPD decreased abnormal mitochondria in diabetic PTCs by restoring autophagy through the suppression of the mTORC1 pathway, which led to improvement in the features of diabetic nephropathy. In addition to renal tubular cells, increased immunohistochemical staining for p62 and p$\mathrm{S} 6 \mathrm{RP}$ in glomerular podocytes was observed. Previous studies have shown that the activation of MTORC1 in glomerular podocytes plays a crucial role in the pathogenesis of diabetic nephropathy $[25,26]$. However, it remains unclear whether mTORC1 activation in podocytes contributes to the suppression of autophagy in diabetes. We propose that reduced albuminuria in WFRs reflects LPD-induced restoration of autophagy induced by the suppression of the mTORC1 pathway in podocytes. Furthermore, the $5.77 \%$ LPD suppressed the mTORC1 pathway and induced autophagy independently of Akt activation in WFRs. However, the $11.46 \%$ LPD did not suppress p-S6RP levels and induce autophagy in the renal cortex. These data indicate that a VLPD (a 5.77\% VLPD, but not an $11.46 \%$ LPD) is needed to suppress the mTORC1 pathway and induce autophagy in the kidney.

An LPD may affect metabolism, on the basis that it represents a high-carbohydrate diet compared with an STD. However, recent studies in mice have shown that ad libitum highcarbohydrate LPDs improve glucose and lipid metabolism and longevity [39]. In the present study, body weight, fat weight, $\mathrm{HbA}_{1 \mathrm{c}}$ and total cholesterol levels were reduced in LPD-fed WFRs compared with STD-fed WFRs. Additionally, the increased levels of FGF21 may be related to LPD-induced metabolic improvement. Laeger et al demonstrated that serum levels of FGF21 specifically increased on exposure to an LPD, regardless of overall calorie intake, in both rodents and humans [40]. FGF21 exerts beneficial effects on energy metabolism, such as increasing glucose uptake into adipose tissue and lowering body weight through increased energy expenditure [41]. Although no differences in fasting FGF21 levels were observed among the four groups, the non-fasting FGF21 levels in both WLRs and LPD-fed WFRs were not reduced; however, they were decreased in WLRs and STD-fed WFRs. Thus, we propose that both food intake reduction and continuous high levels of FGF21 might be associated with lowering blood glucose levels and body and fat weight in WFRs.

In conclusion, this study clearly indicates that VLPD is a beneficial diet therapy for the improvement of advanced diabetic nephropathy by restoring autophagy through the suppression of the MTORC1 pathway in an animal model of type 2 diabetes and obesity. Some patients with advanced diabetic nephropathy rapidly progress to end-stage kidney disease, despite having received adequate multifactorial treatment.
Therefore, a VLPD without malnutrition should be further considered as a clinically relevant means of suppressing the decline in renal function during advanced diabetic nephropathy. However, there may be some issues associated with longterm use of a VLPD in patients, including sarcopenia, frailty or protein-energy wasting, as well as issues associated with adherence to a VLPD. It is also still unknown which amino acids should be restricted for the greatest renoprotection. Therefore, further studies are needed to resolve these points and to develop more useful dietary protocols or replacements for VLPD for advanced stages of diabetic nephropathy.

Acknowledgements We thank A. Takeda-Watanabe, T. Nagai, K. Nitta and Y. Kuroshima (Department of Diabetology and Endocrinology, Kanazawa Medical University, Uchinada, Japan) for their excellent assistance.

Funding This work was financially supported through a Grant-in-Aid for Scientific Research (C) (24591218), a Grant for Promoted Research from Kanazawa Medical University (S2012-4), the Suzuken Memorial Foundation and the Novartis Aging and Geriatric Research Foundation to MK; and by a Grant-in-Aid for Scientific Research (B) (25282028), a Grant-in-Aid for Challenging Exploratory Research (25670414) and a Grant for Collaborative Research from Kanazawa Medical University (C2014-4) to DK. The study was partially supported by a Grant for Promoted Research from Kanazawa Medical University (S2013-13) awarded to KK; and by a Grant for Collaborative Research from Astellas Pharma Inc.

Duality of interest Boehringer Ingelheim, Mitsubishi Tanabe Pharma, Kyowa Hakko Kirin, Taisho Toyama Pharmaceutical Co. and Ono Pharmaceutical Co. contributed to establishing the Division of Anticipatory Molecular Food Science and Technology. The authors declare that there is no duality of interest associated with this manuscript.

Contribution statement MK, KK, SK and DK conceived and designed the experiments. MK, YO, TS, SS and SML contributed to the acquisition of data. MK, YO and TS analysed and interpreted the data. MK, SK, KK and DK contributed to drafting the article. All authors have revised the manuscript critically for important intellectual content and approved the final version to be published. MK, TS and DK are responsible for the integrity of the work as a whole.

\section{References}

1. Packham DK, Alves TP, Dwyer JP et al (2012) Relative incidence of ESRD versus cardiovascular mortality in proteinuric type 2 diabetes and nephropathy: results from the DIAMETRIC (Diabetes Mellitus Treatment for Renal Insufficiency Consortium) database. Am J Kidney Dis 59:75-83

2. Kitada M, Kanasaki K, Koya D (2014) Clinical therapeutic strategies for early stage of diabetic kidney disease. World J Diabetes 5: 342-356

3. Koya D, Haneda M, Inomata S et al (2009) Long-term effect of modification of dietary protein intake on the progression of diabetic nephropathy: a randomised controlled trial. Diabetologia 52:20372045 
4. Johnson DW (2006) Dietary protein restriction as a treatment for slowing chronic kidney disease progression: the case against. Nephrology 11:58-62

5. Robertson L, Waugh N, Robertson A (2007) Protein restriction for diabetic renal disease. Cochrane Database Syst Rev 4, CD002181

6. Klahr S, Levey AS, Beck GJ et al (1994) The effects of dietary protein restriction and blood-pressure control on the progression of chronic renal disease. Modification of Diet in Renal Disease Study Group. N Engl J Med 330:877-884

7. Levey AS, Greene T, Beck GJ et al (1999) Dietary protein restriction and the progression of chronic renal disease: what have all of the results of the MDRD study shown? Modification of Diet in Renal Disease Study Group. J Am Soc Nephrol 10:2426-2439

8. Levey AS, Greene T, Sarnak MJ et al (2006) Effect of dietary protein restriction on the progression of kidney disease: long-term follow-up of the Modification of Diet in Renal Disease (MDRD) Study. Am J Kidney Dis 48:879-888

9. Hansen HP, Tauber-Lassen E, Jensen BR, Parving HH (2002) Effect of dietary protein restriction on prognosis in patients with diabetic nephropathy. Kidney Int 62:220-228

10. Pedrini MT, Levey AS, Lau J, Chalmers TC, Wang PH (1996) The effect of dietary protein restriction on the progression of diabetic and nondiabetic renal diseases: a meta-analysis. Ann Intern Med 124:627-632

11. Nezu U, Kamiyama H, Kondo Y, Sakuma M, Morimoto T, Ueda S (2013) Effect of low-protein diet on kidney function in diabetic nephropathy: meta-analysis of randomised controlled trials. BMJ Open 3

12. Ideura T, Shimazui M, Morita H, Yoshimura A (2007) Protein intake of more than $0.5 \mathrm{~g} / \mathrm{kg} \mathrm{BW} /$ day is not effective in suppressing the progression of chronic renal failure. Contrib Nephrol 155:40-49

13. Seney FD Jr, Persson EG, Wright FS (1987) Modification of tubuloglomerular feedback signal by dietary protein. Am J Physiol 252:F83-F90

14. Sallstrom J, Carlstrom M, Olerud J et al (2010) High-proteininduced glomerular hyperfiltration is independent of the tubuloglomerular feedback mechanism and nitric oxide synthases. Am J Physiol Regul Integr Comp Physiol 299:R1263-R1268

15. Tolins JP, Shultz PJ, Westberg G, Raij L (1995) Renal hemodynamic effects of dietary protein in the rat: role of nitric oxide. J Lab Clin Med 125:228-236

16. Wen SF, Huang TP, Moorthy AV (1985) Effects of low-protein diet on experimental diabetic nephropathy in the rat. J Lab Clin Med 106:589-597

17. Dunger A, Berg S, Kloting I, Schmidt S (1997) Functional alterations in the rat kidney induced either by diabetes or high protein diet. Exp Clin Endocrinol Diabetes 105(Supp1 2):48-50

18. Risdon RA, Sloper JC, De Wardener HE (1968) Relationship between renal function and histological changes found in renal-biopsy specimens from patients with persistent glomerular nephritis. Lancet 2:363-366

19. Gilbert RE, Cooper ME (1999) The tubulointerstitium in progressive diabetic kidney disease: more than an aftermath of glomerular injury? Kidney Int 56:1627-1637

20. Tang SC, Lai KN (2012) The pathogenic role of the renal proximal tubular cell in diabetic nephropathy. Nephrol Dial Transplant 27: 3049-3056

21. Choi AM, Ryter SW, Levine B (2013) Autophagy in human health and disease. N Engl J Med 368:651-662

22. Kume S, Koya D, Uzu T, Maegawa H (2014) Role of nutrientsensing signals in the pathogenesis of diabetic nephropathy. BioMed Res Int 2014:315494
23. Koya D, Kitada M, Kume S, Kanasaki K (2014) Interventions against nutrient-sensing pathways represent an emerging new therapeutic approach for diabetic nephropathy. Clin Exp Nephrol 18: 210-213

24. Sakaguchi M, Isono M, Isshiki $\mathrm{K}$, Sugimoto $\mathrm{T}$, Koya $\mathrm{D}$, Kashiwagi A (2006) Inhibition of mTOR signaling with rapamycin attenuates renal hypertrophy in the early diabetic mice. Biochem Biophys Res Commun 340:296-301

25. Inoki $\mathrm{K}$, Mori $\mathrm{H}$, Wang $\mathrm{J}$ et al (2011) mTORC1 activation in podocytes is a critical step in the development of diabetic nephropathy in mice. J Clin Invest 121:2181-2196

26. Godel M, Hartleben B, Herbach N et al (2011) Role of mTOR in podocyte function and diabetic nephropathy in humans and mice. $\mathrm{J}$ Clin Invest 121:2197-2209

27. Bar-Peled L, Sabatini DM (2014) Regulation of mTORC1 by amino acids. Trends Cell Biol 24:400-406

28. Kitada M, Takeda A, Nagai T, Ito H, Kanasaki K, Koya D (2011) Dietary restriction ameliorates diabetic nephropathy through antiinflammatory effects and regulation of the autophagy via restoration of Sirt1 in diabetic Wistar fatty (fa/fa) rats: a model of type 2 diabetes. Exp Diabetes Res 2011:908185

29. Kume S, Uzu T, Araki S et al (2007) Role of altered renal lipid metabolism in the development of renal injury induced by a high-fat diet. J Am Soc Nephrol 18:2715-2723

30. Yamahara K, Kume S, Koya D et al (2013) Obesity-mediated autophagy insufficiency exacerbates proteinuria-induced tubulointerstitial lesions. J Am Soc Nephrol 24:1769-1781

31. Kume S, Uzu T, Horiike K et al (2010) Calorie restriction enhances cell adaptation to hypoxia through Sirt1-dependent mitochondrial autophagy in mouse aged kidney. J Clin Invest 120:1043-1055

32. Brooks C, Wei Q, Cho SG, Dong Z (2009) Regulation of mitochondrial dynamics in acute kidney injury in cell culture and rodent models. J Clin Invest 119:1275-1285

33. Xu L, Kanasaki M, He J et al (2013) Ketogenic essential amino acids replacement diet ameliorated hepatosteatosis with altering autophagy-associated molecules. Biochim Biophys Acta 1832: $1605-1612$

34. Kroemer G, Marino G, Levine B (2010) Autophagy and the integrated stress response. Mol Cell 40:280-293

35. Archer SL (2013) Mitochondrial dynamics - mitochondrial fission and fusion in human diseases. N Engl J Med 369:2236-2251

36. Higgins GC, Coughlan MT (2014) Mitochondrial dysfunction and mitophagy: the beginning and end to diabetic nephropathy? Br J Pharmacol 171:1917-1942

37. Hartleben B, Godel M, Meyer-Schwesinger C et al (2010) Autophagy influences glomerular disease susceptibility and maintains podocyte homeostasis in aging mice. J Clin Invest 120:10841096

38. Grumati P, Coletto L, Sabatelli P et al (2010) Autophagy is defective in collagen VI muscular dystrophies, and its reactivation rescues myofiber degeneration. Nat Med 16:1313-1320

39. Solon-Biet SM, McMahon AC, Ballard JW et al (2014) The ratio of macronutrients, not caloric intake, dictates cardiometabolic health, aging, and longevity in ad libitum-fed mice. Cell Metab 19:418430

40. Laeger T, Henagan TM, Albarado DC et al (2014) FGF21 is an endocrine signal of protein restriction. J Clin Invest 124:39133922

41. Kharitonenkov A, Shiyanova TL, Koester A et al (2005) FGF-21 as a novel metabolic regulator. J Clin Invest 115:1627-1635 